\title{
A Prospective, Open-Label Short-Term Pilot Study on Modification of the Skin Hydration Status During Treatment With a Sodium-Glucose Cotransporter-2 Inhibitor
}

\author{
Yuji Tezuka · Osamu Sekine · Akiko Hirano - Yukako Hanada · Ikuhisa Nakanishi • \\ Misaki Ariga - Choka Azuma · Yukako Yamamoto · Jun Ito-Kobayashi • Miki Washiyama • \\ Masanori Iwanishi · Miyuki Furuta · Masao Kanamori · Akira Shimatsu $\cdot$ Atsunori Kashiwagi
}

Received: September 8, 2020 / Accepted: October 14, 2020 / Published online: October 27, 2020

(c) The Author(s) 2020

\section{ABSTRACT}

Introduction: Various types of skin lesions with pruritus have been reported in participants of Asian clinical trials on sodium-glucose cotransporter-2 (SGLT2) inhibitors. The aim of this study was to determine whether the diuretic effect of a SGLT2 inhibitor could modify skin hydration status in patients with type 2 diabetes mellitus.

Methods: A prospective, short-term, open-label, two-parallel-arm, pilot study was conducted. Eligible patients were assigned to either a SGLT2 inhibitor (50 mg ipragliflozin once daily) group or to a dipeptidyl peptidase- 4

Y. Tezuka · O. Sekine - C. Azuma · Y. Yamamoto . J. Ito-Kobayashi · M. Washiyama - M. Iwanishi . A. Shimatsu · A. Kashiwagi $(\square)$

Department of Diabetes and Endocrinology, Kusatsu General Hospital, 1660 Yabase, Kusatsu, Shiga 5258585, Japan

e-mail: kashiwagi@kusatsu-gh.or.jp

A. Hirano · Y. Hanada · I. Nakanishi · M. Ariga R\&D Department, Sunstar Inc., 3-1 Asahi-machi, Takatsuki, Osaka 569-1195, Japan

M. Furuta

Department of Dermatology, Kusatsu General Hospital, 1660 Yabase, Kusatsu, Shiga 525-8585, Japan

M. Kanamori

College of Health and Sport Science, Ritsumeikan University, 1-1-1 Noji Higashi, Kusatsu, Shiga 5258577, Japan inhibitor (50 mg sitagliptin once daily) group (control). The biophysical characteristics of the skin were measured and blood chemistry tests were run in all participants 1 day prior to medication initiation (pre-treatment values) and 14 days thereafter (post-treatment values).

Results: Fourteen patients were enrolled in the study, of whom eight were in the ipragliflozin group and six in the sitagliptin group. Compared to the pre-treatment values, the glycated hemoglobin (HbA1c) levels were slightly but significantly reduced in the ipragliflozin group $(p=0.02)$, but the changes in HbA1c from the pre-treatment to post-treatment time points did not significantly differ between the two treatment groups. Serum 3-hydroxy butyrate levels were significantly higher in the ipragliflozin group than in the sitagliptin group $(p<0.02)$. Neither electrical capacitance nor electrical conductance of the stratum corneum (SC), parameters that reflect skin water content, was reduced by 14 days of ipragliflozin treatment; similarly, no changes in these parameters were found in the sitagliptin control group. There was also no difference in the changes in water barrier function of the SC between the two treatment groups. There was a significant linear correlation $(p<0.01)$ in skin water content at pre-treatment and that 14 days after treatment with each drug, respectively.

Conclusion: Ipragliflozin treatment for 14 days did not significantly affect the skin hydration status in patients with well-controlled type 2 
diabetes mellitus.Keywords: Ipragliflozin; Prospective study; SGLT2 inhibitor; Skin dehydration; Skin disorder; Type 2 diabetes mellitus

\section{Key Summary Points}

Why carry out this study?

Sodium-glucose cotransporter-2 (SGLT2) inhibitors increase both urinary glucose excretion and urine volume in both healthy nondiabetic and diabetic subjects.

Urinary glucose depletion, osmotic diuresis, polyuria/pollakiuria and volume depletion may cause genital infections and skin disorders, which are more frequent with SGLT2 inhibitor treatment than with placebo.

We examined whether the diuretic effect of a SGLT2 inhibitor would modify the skin hydration status in patients with type 2 diabetes mellitus (T2DM), similar to dry skin in patients treated with diuretics.

\section{What was learned from the study?}

Fourteen days of ipragliflozin treatment did not significantly affect primary endpoints of the skin hydration status, which were measured on three standard types of devices (Corneometer ${ }^{\circledR}$, Skicon ${ }^{\circledR}$ and Tewameter ${ }^{\circledR}$ ) in patients with wellcontrolled T2DM.

The results suggest that the observed small change in skin water content due to exposure to SGLT2 inhibitors might be compensated for during a short-term treatment in patients with well-controlled T2DM.

\section{DIGITAL FEATURES}

This article is published with digital features, including a summary slide, to facilitate understanding of the article. To view digital features for this article, go to https://doi.org/10. 6084/m9.figshare.13084745.

\section{INTRODUCTION}

Sodium-glucose cotransporter-2 (SGLT2) inhibitors are oral antihyperglycemic agents that inhibit the reabsorption of filtrated glucose in proximal renal tubular cells. Increases in both urinary glucose excretion and urine volume associated with the use of SGLT2 inhibitors have been reported in both healthy nondiabetic and diabetic subjects [1]. Consequently, treatment-emergent adverse events (TEAEs) related to urinary glucose depletion, osmotic diuresis, polyuria/pollakiuria and volume depletion may cause genital infection and skin disorders, which are more frequent in persons treated with ipragliflozin, a next-generation SGLT2 inhibitor, than in those on placebo [2]. On the other hand, SGLT2 inhibitors have many benefits, such as reducing various factors related to the risk of cardiometabolic disease developmentwith the exception of serum low-density lipoprotein cholesterol and serum non-highdensity lipoprotein cholesterol levels [3]through multiple hemodynamic and metabolic biological effects $[4,5]$. Indeed, SGLT2 inhibitors have been shown to be associated with greater reductions in cardiovascular events, hospitalizations for heart failure and all-cause mortality in patients with type 2 diabetes mellitus (T2DM) with high cardiovascular risk profiles as compared with placebo [6-8].

Various types of skin lesions with pruritus have been reported in participants in Asian clinical trials on SGLT2 inhibitors, with the incidence rate being approximately $2-3 \%$, and higher in the ipragliflozin treatment group versus placebo group $[2,9,10]$. In addition, skin lesions are usually observed in studies on SGLT2 inhibitors within 14 days after treatment initiation [11]. SGLT2 inhibitors have been reported to transiently increase the urine volume of patients on the first day after medication initiation, with a return to pre-treatment (baseline) levels thereafter $[12,13]$. Thus, skin disorders may be related to skin dehydration due to 
glucosuria and osmotic diuresis, in a manner comparable to diuretic use increasing the incidence of dry skin [14]. The volume depletion that occurs during SGLT2 inhibitor treatment is similar to that occurring with the use of classical diuretics and is related to a reduction in the interstitial volume, although unlike with classical diuretics, the intravascular volume is well maintained in treatments with SGLT2 inhibitors [15]. Thus, although little is known about the changes in skin hydration status following treatment with SGLT2 inhibitors, we hypothesize that the skin disorders may be at least partly related to dehydration of the subcutaneous tissue. The authors of one study reported that skin hydration status was not significantly different between patients with diabetes and healthy people [16], but results from other studies suggest that poor glycemic control may be associated with dry skin $[14,17]$.

Based on current evidence, we considered it useful to compare post-treatment changes in blood glucose between patients with T2DM initiating an SGLT2 inhibitor and those initiating a dipeptidyl peptidase-4 (DPP-4) inhibitor as a control. We therefore conducted a prospective, short-term, open-label, two-parallel-arm, pilot study using ipragliflozin, the first SGLT2 inhibitor to be approved in Japan, and sitagliptin, a DPP-4 inhibitor, as an active control that achieves comparable glycemic control. Our aim was to evaluate changes in skin hydration 14 days after the intiation of the treatments in patients with T2DM.

\section{METHODS}

\section{Study Population}

Eligible subjects were female and male patients with T2DM with the following characteristics: aged $\geq 50$ years and $<75$ years; good glycemic control, with glycated hemoglobin (HbA1c) (National Glycohemoglobin Standardization Program [NGSP]; http://www.ngsp.org/) $\geq 6.5 \%$ and $<8.0 \%$ and fluctuations within $0.3 \%$ in the 2 months immediately preceding enrollment; and currently treated, or not, with an oral hypoglycemic medicine (only metformin $\leq$
$500 \mathrm{mg} /$ day was allowed). Diabetic patients were excluded if they were pregnant; had atopic dermatitis, eczema, psoriasis or other dermatologic disorders, or suntans, during the study period; had complicating foot disorders, such as injuries, ulcers or gangrene; had complications of severe liver dysfunction, severe kidney dysfunction or myocardial infarction; had a history of peripheral artery disease or severe complications of peripheral edema or peripheral circulatory failure; or used body cream daily on the part of the body where skin hydration was measured.

\section{Procedures}

This double-arm, exploratory study was conducted to investigate changes in skin hydration in Japanese patients with T2DM treated with either ipragliflozin or sitagliptin. We also examined the changes in biochemical parameters related to dehydration. The study was conducted between October and March in 2017, 2018 and 2019, respectively, in order to eliminate seasonal changes in the skin's water content [18]. Eligible patients were assigned to either an SGLT2 inhibitor group (50 mg ipragliflozin once daily) or a DPP-4 inhibitor group (50 mg sitagliptin once daily; active control group) based on the inclusion criteria, not by randomization but by the treating physician's choice because we thought it would be difficult to recruit participants to a randomized controlled trial in our hospital. The patients in the ipragliflozin group were instructed to increase their normal daily intake of water by $500 \mathrm{ml}$ to prevent dehydration. Daily medication adherence was recorded in a diary. Each patient was followed up to confirm compliance to daily medicinal dose provided. No prescription medicines were changed for any participants during the study period. Participants were required not to use body cream on the part of the body where skin hydration was measured.

The treatment was maintained for 14 days in both groups. Fasting blood samples for laboratory tests were collected in the morning 1 day before starting the medication (pre-treatment) 
and on the last day of the treatment (posttreatment).

\section{Noninvasive Measurements of the Hydration Status in the Stratum Corneum}

The biophysical characteristics of the skin were measured on the anterior surface of the forearm and extensor surface of the lower leg using the following noninvasive biophysical methods in the morning with subjects in the fasting state. Dehydration of the stratum corneum (SC) was evaluated using Corneometer CM $825^{\circledR}$ (Courage-Khazaka, Cologne, Germany) and Skicon$200^{\circledR}$ skin conductance meters (I.B.S. Co., Hamamatsu, Japan). The Corneometer measured the skin's electrical capacitance to determine the water content of the SC [19], while the Skicon meter measured the skin's electrical conductance as an additional index of water content [20]. Transepidermal water loss (TEWL), a measurement of the water barrier function of the SC, was determined using the Tewameter TM300 ${ }^{\circledR}$ measuring device (Courage-Khazaka, Cologne, Germany) [21]. The water content and water barrier function values were expressed as the mean of five and three serial measurements, respectively, and the mean values of both sides of the forearm and lower leg were calculated, respectively. The preliminary analysis showed that the coefficient of variation (CV) of the raw data on the lower legs of 11 healthy subjects, as measured by the Corneometer, Skicon meter and Tewameter, were 14.4, 20.1 and $8.1 \%$, respectively.

Before initiating the measurements, participants were acclimated to a preadjusted temperature of $20 \pm 2{ }^{\circ} \mathrm{C}$ and relative humidity of $50 \pm 5 \%$ for $20 \mathrm{~min}$ in a private room.

The primary endpoints of the study were changes in the hydration status of the SC as assessed by the Corneometer, Skicon and Tewameter.

\section{Ethics}

The study was conducted in accordance with the ethical principles of the Declaration of
Helsinki (revised October 2013) and with the Ethical Guidelines for Medical and Health Research Involving Human Subjects, and the Conflict-of-Interest Management Rules for Clinical Studies of the Kusatsu General Hospital (Kusatsu, Japan). The Institutional Review Board at the Kusatsu General Hospital approved the study protocol (approval date: 8 Sep 2017; Approval no. 2017-1011-02). All participants provided written informed consent before participating in the study.

\section{Statistical Analysis}

Continuous variables are shown as the median and interquartile range. Categorical variables are presented as numbers and percentages. We compared categorical variables using Fisher's exact test and compared continuous variables using the Wilcoxon rank-sum test due to the small sample size. The Wilcoxon signed-rank test was also used to demonstrate differences between the parameters pre-treatment and 14 days post-treatment. We used Pearson's correlation coefficients to measure the association of the changes in hydration parameters at both of these time points. We could not appropriately calculate the sample size because of the wide inter- and intra-individual variations in the measurements obtained by the instruments used for estimating skin hydration.

JMP version 11 software (SAS Institute, Cary, NC, USA) was used for statistical analyses. Twosided $p$ values $<0.05$ were considered to indicate significance.

\section{RESULTS}

\section{Subjects}

Fourteen patients were enrolled in the study, eight in the ipragliflozin group and six in the sitagliptin group. Data from the forearm of one female patient in the ipragliflozin group were excluded from the analysis because she used body cream on her forearm every day; however, data measured at the extensor surface of her lower leg were included for analysis. 
The baseline (pre-treatment) demographics and characteristics of the patients are given in Table 1. All baseline characteristics, including

Table 1 Baseline characteristics and co-morbidities of the subjects

\begin{tabular}{|c|c|c|c|}
\hline $\begin{array}{l}\text { Baseline } \\
\text { characteristics } \\
\text { and co- } \\
\text { morbidities }\end{array}$ & $\begin{array}{l}\text { Ipragliflozin } \\
\text { group } \\
(n=8)\end{array}$ & $\begin{array}{l}\text { Sitagliptin } \\
\text { group } \\
(n=6)\end{array}$ & $p$ value \\
\hline Female & $4(50.0 \%)$ & $3(50.0 \%)$ & 1.00 \\
\hline Age (years) & $68(66,70)$ & $69(60,72)$ & 1.00 \\
\hline Body weight (kg) & $\begin{array}{c}66.2(56.4 \\
72.5)\end{array}$ & $\begin{array}{c}69.9(56.4 \\
76.2)\end{array}$ & 0.65 \\
\hline $\begin{array}{l}\text { Body mass index } \\
\qquad\left(\mathrm{kg} / \mathrm{m}^{2}\right)\end{array}$ & $\begin{array}{c}24.5(22.7 \\
28.1)\end{array}$ & $\begin{array}{c}25.3(24.1 \\
26.7)\end{array}$ & 0.65 \\
\hline $\begin{array}{l}\text { Systolic blood } \\
\text { pressure } \\
(\mathrm{mmHg})\end{array}$ & $\begin{array}{c}133(119 \\
140)\end{array}$ & $\begin{array}{c}125(117, \\
138)\end{array}$ & 0.48 \\
\hline $\begin{array}{l}\text { Diastolic blood } \\
\text { pressure } \\
(\mathrm{mmHg})\end{array}$ & $72(68,75)$ & $75(67,85)$ & 0.65 \\
\hline Pulse rate (bpm) & $61(54,68)$ & $68(64,78)$ & 0.22 \\
\hline $\begin{array}{l}\text { Diabetes duration } \\
\text { (years) }\end{array}$ & $2.5(0.5,4.8)$ & $3(0,9.3)$ & 0.94 \\
\hline Microalbuminuria & $1(12.5 \%)$ & $1(16.7 \%)$ & 1.00 \\
\hline Hypertension & $5(62.5 \%)$ & $2(33.3 \%)$ & 0.59 \\
\hline Dyslipidemia & $6(75 \%)$ & $4(66.7 \%)$ & 1.00 \\
\hline HbAlc (\%) & $6.8(6.3,7.2)$ & $\begin{array}{c}6.2(6.2 \\
7.0)\end{array}$ & 0.43 \\
\hline \multicolumn{4}{|l|}{ Medication } \\
\hline Metformin & $2(25.0 \%)$ & $4(66.7 \%)$ & 0.28 \\
\hline $\begin{array}{l}\text { Antihypertensive } \\
\text { drug }\end{array}$ & $4(50.0 \%)$ & $2(33.3 \%)$ & 0.63 \\
\hline Statin & $6(75 \%)$ & $3(50.0 \%)$ & 0.58 \\
\hline
\end{tabular}

Categorial data are presented as the number with the percentage in parentheses; continuous data are presented as the median with the interquartile range (IQR; 25\%, 75\%) in parentheses

HbAlc Glycated hemoglobin age, sex, body mass index, HbA1c and blood pressure, were similar between the two treatment groups. In addition, all patients had diabetes for a short period of time and were maintaining a good glycemic status. No diuretics were prescribed for any participant. The biophysical characteristics of the skin and blood chemistry of the patients in both groups were compared, with the results showing no difference in these parameters at baseline between the groups (Table 2).

\section{Treatment Effects on the Blood Chemistry}

The changes in blood chemistry measures in this study cohort of 14 patients with well-controlled T2DM over the study period are shown in Table 2 . At the post-treatment time point, HbA1c levels were slightly but significantly reduced in the ipragliflozin treatment group $(p=0.02)$ compared the pre-treatment values and the fasting blood glucose levels showed a tendency to be reduced $(p=0.10)$. However, neither HbA1c nor fasting glucose levels were significantly reduced by 14 days of sitagliptin treatment. Changes in HbA1c at 14 days after initiation of the drug treatment did not differ between the two treatment groups, but the increases in serum 3-hydroxybutyric acid levels were significantly higher in the ipragliflozin group than in the sitagliptin group $(p=0.02)$.

\section{Effect of Ipragliflozin on the Biophysical Status of the Skin}

Changes in the electrical capacitance and electrical conductance of the skin, assessed using the Corneometer and Skicon meter, respectively, as a marker of SC water content, were not significantly different between the ipragliflozin and sitagliptin treatment groups. Changes in TEWL, measured using the Tewameter, were also not significantly different between the two treatment groups (Table 2). 


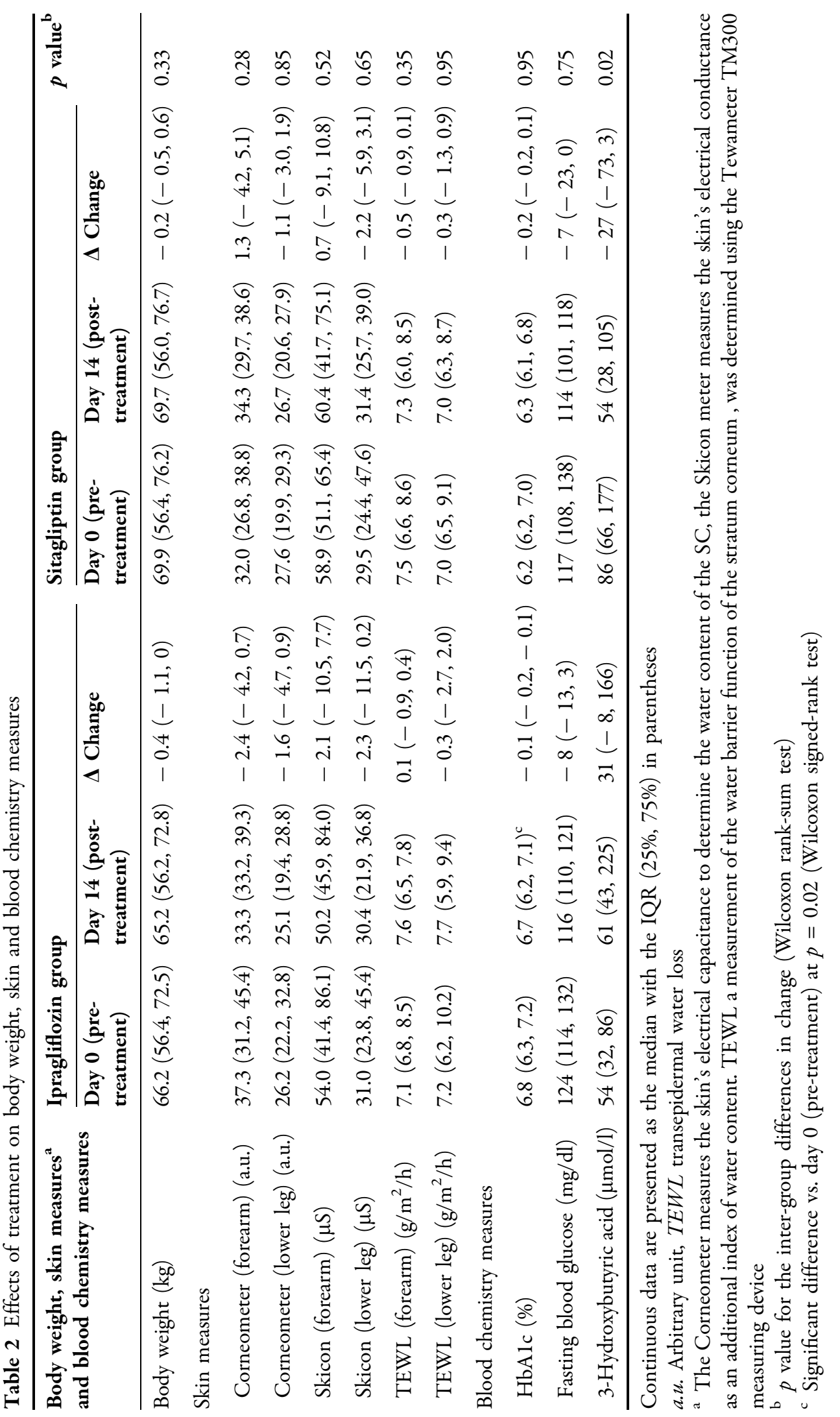




\section{Correlation Between the SC Water Content and Tewl of SC Between Before and After Treatment}

Scatter plots of the parameters of skin electrical capacitance, skin electrical conductance, and water barrier function, which were analyzed using the combined data from both the forearms and lower legs before and after the treatments, are shown in Fig. 1a, b, c, respectively. There were significant $(p<0.01)$ linear correlations between pre-treatment and post-treatment measures of electrical capacitance (Fig. 1a) and electrical conductance (Fig. 1c) in both the ipragliflozin and sitagliptin treatment groups. The TEWL was significantly $(p<0.01)$ linearly correlated in the sitagliptin group, but not in the ipragliflozin group ( $p=0.12$ ) (Fig. 1c).

\section{Adverse Events}

No skin disorders occurred in either treatment group. One woman in the ipragliflozin group experienced complicated vaginitis without drug interruption. The patient quickly recovered from the vaginitis after the end of the ipragliflozin treatment and the appropriate antibiotic therapy.

\section{DISCUSSION}

This prospective clinical study is the first study to examine the effect of an SGLT2 inhibitor on the skin hydration status in patients with T2DM. Asian clinical trials have reported that the incidence rate of various types of skin disorders with pruritis is about $2-3 \%$ in patients treated with SGLT2 inhibitors, and higher than that of the respective control groups $[2,9,10]$. One explanation is that the skin disorders may be related to a form of skin water loss, such as senile xerosis. However, in our study, the changes in SC water content measured by a Corneometer and Skicon meter, as well as the SC water barrier function measured by a Tewameter, in both the forearms and lower legs were not decreased following 2 weeks of treatment with a SGLT2 inhibitor, and the results are similar to those obtained in the sitagliptin treatment. Interestingly, the values of SC water (a)

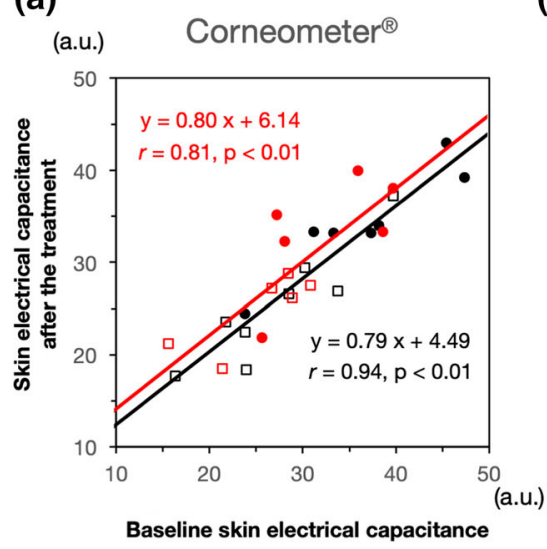

(b)

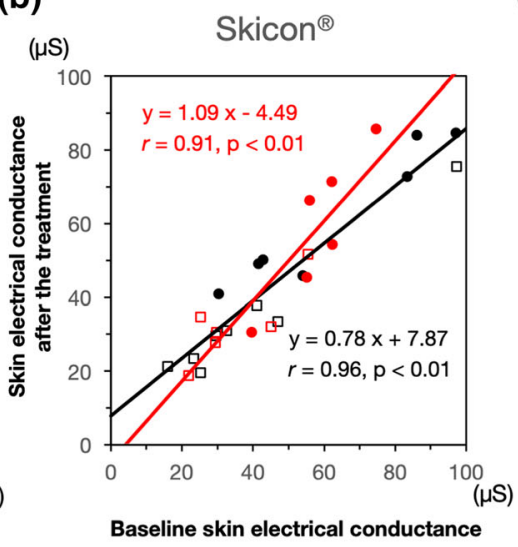

- ipragliflozin (forearm) $\square$ ipragliflozin (lower leg) ipragliflozin (forearm)
sitagliptin (forearm)
a sitagliptin (lower leg) (c)

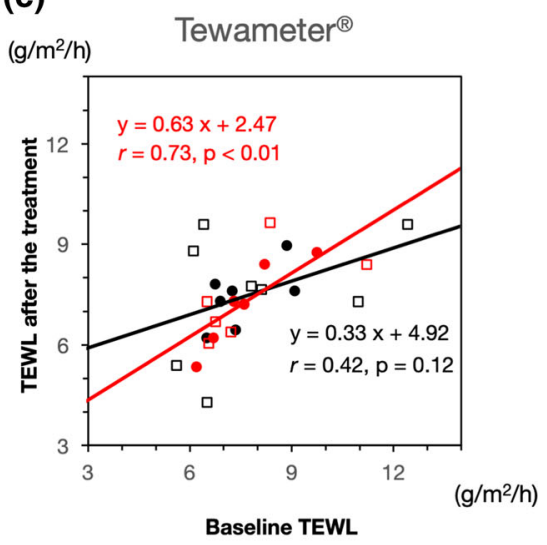

Fig. 1 Scatter plots of the parameters of skin water content. a Electrical capacitance measured with a Corneometer, $\mathbf{b}$ electrical conductance measured with a Skicon meter, $\mathbf{c}$ water barrier function based on transepidermal water loss (TEWL) measured with a Tewameter. All parameters were analyzed using the combined data for forearms (Filled black circle, ipragliflozin group; filled red circle, sitagliptin group) and lower legs (open black square, ipragliflozin group; open red square, sitagliptin group) before ( $x$ axis) and after $(y$-axis) the respective drug treatments. Correlations and linear regression lines between the water content values of the stratum corneum and TEWL were analyzed individually for the ipragliflozin $(n=15)$ and sitagliptin $(n=12)$ treatment groups, a.u. Arbitrary units 
content in the forearms were higher than those in the lower legs, but the TEWL of the SC in the forearms was similar to the value in the lower legs. In addition, although the baseline SC water content varied broadly among individual patients (with a maximum difference of approximately fourfold), the SC water contents measured by the two methods were significantly linearly correlated with the values measured before and after treatments, respectively. These results suggest that treatment with ipragliflozin adequately compensates for the loss of SC water content even under a mild dehydration condition or that the individual variation is larger than the mild skin dehydration effects, if any, during treatment with ipragliflozin.

Previous clinical studies have shown that both SC water content and TEWL are modified by the measurement conditions and by patient characteristics, such as room temperature, humidity, seasonal variation, age and glycemic control status in patients $[17,18]$. In the present study, the baseline glycemic control and changes in HbA1c and fasting blood glucose after each drug treatment were confirmed to be comparable between the two groups. Moreover, the ipragliflozin treatment slightly but significantly reduced HbA1c values $(p=0.02)$ compared with the baseline value. In addition, serum 3-hydroxybutyric acid levels $(p=0.02)$ were significantly increased by ipragliflozin treatment as compared with the changes in the sitagliptin treatment group. These results indicate that the metabolic effects of ipragliflozin treatment were identified in the laboratory data. It is also worth noting that all patients kept a diary to confirm that they took $100 \%$ of the provided medicine. Thus, these results suggest that ipragliflozin treatment for 14 days did not induce any changes in the parameters of skin hydration status in the present group of patients with well-controlled T2DM.

Several limitations of this study may explain why the SC water content and water barrier function were not significantly altered in the ipragliflozin group. First, the amount of fluid consumed during the study period was not recorded, although all patients were instructed to drink $500 \mathrm{ml}$ daily more than their habitual fluid intake and the changes in body weight were not different after the drug treatment between the two groups. Second, the SC might not be sufficiently dehydrated to be affected by a 14-day ipragliflozin treatment. Longer treatment with ipragliflozin may be required to evaluate the drug's effects on the skin hydration status. Third, SGLT2 inhibitors continuously increase urinary glucose excretion even in healthy nondiabetic subjects [22], and increased osmotic diuresis might indicate systemic body water loss and loss of skin water content. Although urinary glucose excretion depends on plasma glucose levels [1], the average glycemic levels of the present participants were wellcontrolled, with a median HbA1c of $<7.0 \%$ in both treatment groups. Furthermore, urine volume and natriuresis have been reported to be compensated for with increasing time after ipragliflozin treatment $[12,13]$. Consequently, it may be speculated that the small change in skin water content might have been compensated for during the 14-day treatment in these patients with well-controlled T2DM. Fourth, our results showed that there were large interindividual variations in SC water content measured by either a Corneometer or Skicon meter and water barrier function measured by a Tewameter, even among our homogeneous group of patients with T2DM with good glycemic control at baseline. The coefficient variation of each skin hydration measurement was also relatively high. Thus, it is possible that our study design could not discriminate small changes in water hydration status by the treatment of ipragliflozin above the measurement variations. Therefore, a a long-term clinical trial is warranted to evaluate the effects of SGLT2 inhibitor treatment on the water content and TEWL of the SC in patients with T2DM with longer duration of diabetes and higher HbA1c levels .

\section{CONCLUSION}

In this prospective, short-term, open-label exploratory study, we did not observe significant changes of skin hydration status, as measured using current standardized methods for evaluation of water content and barrier function of the 
skin, during a 14-day treatment with ipragliflozin in patients with well-controlled T2DM.

\section{ACKNOWLEDGEMENTS}

We thank the participants of the study.

Funding. This study was in part supported by Sunstar Inc. The Rapid Service Fee was funded by the authors.

Authorship. All named authors meet the International Committee of Medical Journal Editors (ICMJE) criteria for authorship for this article, take responsibility for the integrity of the work as a whole, and have given their approval for this version to be published.

Disclosures. AK has acted as a medical consultant for Sunstar Inc. and has received consulting fees. $\mathrm{AH}, \mathrm{YH}, \mathrm{IN}$, and MA are employees of Sunstar Inc. The remaining authors, YT, OS, CA, YY, JIK, MW, MI, MF, MK and AS, have nothing to disclose.

Compliance with Ethics Guidelines. The study was conducted in accordance with the ethical principles of the Declaration of Helsinki (revised October 2013) and with the Ethical Guidelines for Medical and Health Research Involving Human Subjects, and the Conflict-ofInterest Management Rules for Clinical Studies of the Kusatsu General Hospital (Kusatsu, Japan). The Institutional Review Board at the Kusatsu General Hospital approved the study protocol (approval date: 8 Sep 2017; approval no. 2017-1011-02). All participants provided written informed consent before participating in the study.

Data Availability. The datasets generated during and/or analyzed during the current study are available from the corresponding author on reasonable request.

Open Access. This article is licensed under a Creative Commons Attribution-NonCommercial 4.0 International License, which permits any non-commercial use, sharing, adaptation, distribution and reproduction in any medium or format, as long as you give appropriate credit to the original author(s) and the source, provide a link to the Creative Commons licence, and indicate if changes were made. The images or other third party material in this article are included in the article's Creative Commons licence, unless indicated otherwise in a credit line to the material. If material is not included in the article's Creative Commons licence and your intended use is not permitted by statutory regulation or exceeds the permitted use, you will need to obtain permission directly from the copyright holder. To view a copy of this licence, visit http://creativecommons.org/licenses/by$\mathrm{nc} / 4.0 /$.

\section{REFERENCES}

1. DeFronzo RA, Hompesch M, Kasichayanula S, et al. Characterization of renal glucose reabsorption in response to dapagliflozin in healthy subjects and subjects with type 2 diabetes. Diabetes Care. 2013;12(36):3169-76.

2. Kashiwagi A, Shestakova MV, Ito Y, et al. Safety of ipragliflozin in patients with type 2 diabetes mellitus: polled analysis of phase II/III/IV clinical trials. Diabetes Ther. 2019;10(6):2201-17. https://doi.org/ 10.1007/s13300-019-00699-8.

3. Kashiwagi A, Sakatani T, Nakamura I, et al. Improved cardiometabolic risk factors in Japanese patients with type 2 diabetes treated with ipragliflozin: a pooled analysis of six randomized, placebocontrolled trials. Endocr J. 2018;65:691-705.

4. Kashiwagi A, Maegawa H. Metabolic and hemodynamic effects of sodium dependent glucose cotransporter 2 inhibitors on cardio-renal protection in the treatment of patients with type 2 diabetes mellitus. J Diabetes Investig. 2017;8:416-27.

5. Kashiwagi A, Araki S, Maegawa H. Sodium glucose transporter 2 inhibitors represent a paradigm shift in the prevention of heart failure in type 2 diabetes mellitus. J Diabetes Investig. 2020. https://doi.org/ 10.1111/jdi.13329.

6. Zinman B, Wanner C, Lachin JM, et al. Empagliflozin, cardiovascular outcomes, and mortality in type 2 diabetes. N Engl J Med. 2015;373:2117-28. 
7. Neal B, Perkovic V, Mahaffey KW, et al. Canagliflozin and cardiovascular and renal events in type 2 diabetes. N Engl J Med. 2017;377:644-57.

8. Wiviott SD, Raz I, Bonaca MP, et al. Dapagliflozin and cardiovascular outcomes in type 2 diabetes. N Engl J Med. 2019;380:347-57.

9. Yokote K, Terauchi Y, Nakamura I, et al. Real-world evidence for the safety of ipragliflozin in elderly Japanese patients with type 2 diabetes mellitus (STELLA-ELDER): final results of a post-marketing surveillance study. Expert Opin Pharmacother. 2016;17:1995-2003.

10. Lu CH, Min KW, Chuang LM, et al. Efficacy, safety, and tolerability of ipragliflozin in Asian patients with type 2 diabetes mellitus and inadequate glycemic control with metformin: results of a phase 3 randomized, placebo-controlled, double-blind, multicenter trial. J Diabetes Investig. 2016;7: 366-73.

11. Yabe D, Nishikino R, Kaneko M, et al. Short-term impacts of sodium/glucose co-transporter 2 inhibitors in Japanese clinical practice: considerations for their appropriate use to avoid serious adverse events. Expert Opin Drug Saf. 2015;14:795-800.

12. Tanaka $\mathbf{H}$, Takano $\mathrm{K}$, Iijima $\mathrm{H}$, et al. Factors affecting canagliflozin-induced transient urine volume increase in patients with type 2 diabetes mellitus. Adv Ther. 2017;34:436-51.

13. Hallow KM, Helmlinger G, Greasley PJ, et al. Why do SGLT2 inhibitors reduce heart failure hospitalization? A differential volume regulation hypothesis. Diabetes Obes Metab. 2018;20:479-87.

14. Mekic S, Jacobs LC, Gunn DA, et al. Prevalence and determinants for xerosis cutis in the middle-aged and elderly population: a cross-sectional study. J Am Acad Dermatol. 2019;81(4):963-9.e2.
15. Lumbers Heerspink HJ, deZeeuw D, Wie L, Leslie B, List J. Dapagliflozin a glucose-regulating drug with diuretic properties in subjects with type 2 diabetes. Diabetes Obes Metab. 2013;15:853-62.

16. Seirafi H1, Farsinejad K, Firooz A, et al. Biophysical characteristics of skin in diabetes: a controlled study. J Eur Acad Dermatol Venereol. 2009; 23: 146-9.

17. Sakai S, Kikuchi K, Satoh J, et al. Functional properties of the stratum corneum in patients with diabetes mellitus: similarities to senile xerosis. $\mathrm{Br} \mathrm{J}$ Dermatol. 2005;153:319-23.

18. du Plessis J1, Stefaniak A, Eloff F, et al. International guidelines for the in vivo assessment of skin properties in non-clinical settings: part 2. transepidermal water loss and skin hydration. Skin Res Technol. 2013;19:265-78.

19. Clarys P, Clijsen R, Taeymans J, et al. Hydration measurements of the stratum corneum: comparison between the capacitance method (digital version of the Corneometer CM 825®) and the impedance method (Skicon-200EX®). Skin Res Technol. 2012;18:316-23.

20. Tagami H, Ohi M, Iwatsuki K, et al. Evaluation of the skin surface hydration in vivo by electrical measurement. J Invest Dermatol. 1980;75:500-7.

21. Rogiers V, EEMCO Group. EEMCO guidance for the assessment of transepidermal water loss in cosmetic sciences. Skin Pharmacol Appl Skin Physiol. 2001;14:117-28.

22. Sha S, Devineni D, Ghosh A, et al. Canagliflozin, a novel inhibitor of sodium glucose co-transporter 2 , dose dependently reduces calculated renal threshold for glucose excretion and increase urinary glucose excretion in healthy subjects. Diabetes Obes Metab. 2011;13:669-72. 